\title{
Kaempferol Alleviates Angiotensin II-Induced Cardiac Dysfunction and Interstitial Fibrosis in Mice
}

\author{
Yuan Liua Lu Gao Sen Guo $^{\mathrm{a}}$ Yuzhou Liu ${ }^{\mathrm{a}} \quad$ Xiaoyan Zhao ${ }^{\mathrm{a}} \quad$ Ran $\mathrm{Li}^{\mathrm{a}}$ \\ Xiaofei Yan ${ }^{a}$ Yunpeng Li $^{a}$ Shuai Wang ${ }^{b}$ Xiaoyu Niu ${ }^{a}$ Liantao Yao \\ Yanzhou Zhang ${ }^{\mathrm{a}}$ Ling Lia Haibo Yang ${ }^{\mathrm{a}}$ \\ aDepartment of Cardiology, the First Affiliated Hospital of Zhengzhou University, Zhengzhou, \\ bDepartment of Ultrasound, the First Affiliated Hospital of Zhengzhou University, Zhengzhou, China
}

\section{Key Words}

Kaempferol - Cardiac fibrosis - Endothelial-to-mesenchymal transition - Endothelium • Angiotensin II

\begin{abstract}
Background/Aims: Endothelial-to-mesenchymal transition (EndMT) is a mechanism that promotes cardiac fibrosis induced by Angiotensin II (AngII). Kaempferol (KAE) is a monomer component mainly derived from the rhizome of Kaempferia galanga L. It shows antiinflammatory, anti-oxidative, anti-microbial and anti-cancer properties, which can be used in the treatment of cancer, cardiovascular diseases, infection, etc. But, its effects on the development of cardiac remodelling remain completely unknown. The aim of the present study was to determine whether KAE attenuates cardiac hypertrophy induced by angiotensin II (Ang II) in cultured neonatal rat cardiac myocytes in vitro and cardiac hypertrophy induced by AngII infusion in mice in vivo. Methods: Male wild-type mice aged 8-10 weeks with or without KAE were subjected to AngII or saline, to induce fibrosis or as a control, respectively. Morphological changes, echocardiographic parameters, histological analyses, and hypertrophic markers were also used to evaluate hypertrophy. Results: KAE prevented and reversed cardiac remodelling induced by AngII. The KAE in this model exerted no basal effects but attenuated cardiac fibrosis, hypertrophy and dysfunction induced by AngII. Both in vivo and in vitro experiments demonstrated that Ang II infusion or TGF- $\beta$ induced EndMT can be reduced by KAE and the proliferation and activation of cardiac fibroblasts (CFs) can be inhibited by KAE. Conclusions: The results suggest that KAE prevents and reverses ventricular fibrosis and cardiac dysfunction, providing an experimental basis for clinical treatment on ventricular fibrosis.
\end{abstract}

Y. Liu, L. Gao and S. Guo are co-first authors.

Haibo Yang

KARGER
Department of Cardiology, The First Affiliated Hospital of Zhengzhou University

No.1 Jianshe East Road, Zhengzhou, (China)

Tel. +86 13526477206, E-Mail yhb160320@163.com 


\section{Introduction}

Cardiac fibrosis, which is caused by the accumulation of excessive extracellular matrix (ECM) proteins within the myocardium, is associated with decreased microvasculature and increased stiffness [1-4]; The ECM is predominantly produced by myofibroblasts, which may be trans-differentiated from cardiac fibroblasts [5]. Several previous studies have determined that aside from resident fibroblasts, cardiac fibroblasts are a heterogeneous cell population involved in the development of cardiac disease and are likely derived from various distinct tissue niches, including endothelial cells [6], epicardial cells [7], bone marrow (BM)derived fibrocytes [8,9], monocytes and pericytes [10]. Although anti-fibrotic therapies may improve the long-term prognosis of cardiovascular disease patients, there are no specific drugs available to treat these diseases.

The Endothelial-to-mesenchymal transition (EndMT) is a mechanism that promotes cardiac fibrosis during aortic banding $(\mathrm{AB})$-induced pressure overload. It was first discovered by Zeisberg [6]. The transition process is characterized by the loss of endothelial cellular characteristics and the acquisition of a mesenchymal phenotype. The cells acquired from endothelial cells via the EndMT mechanism account for $27-35 \%$ among fibroblasts, and ventricular remodelling and cardiac function can be dramatically improved by inhibiting EndMT. Widyantoro et al. [11] demonstrated that endothelial cells-derived endothelin-1 (ET1) promoted cardiac fibrosis in the diabetic heart via the stimulation of the EndMT. Murdoch et al [12] revealed that endothelial NADPH oxidase-2 promoted interstitial cardiac fibrosis and diastolic dysfunction-induced by angiotensin II (AngII) via the important mechanism of the EndMT. As the molecular mechanisms underlying its development have not been clearly elucidated, a better understanding of the factors that regulate cardiac fibrosis may reveal potential therapeutic targets useful for treating cardiac remodelling.

Kaempferol (C15H1006) is a flavonoid mainly derived from the rhizome of Kaempferia galanga L, which is not only a traditional medicine but also a famous flavour and condiment $[13,14]$. The previous studies have confirmed its properties of anti-inflammation, antioxidation, anti-microbes, and anti-cancer, which can be used in the treatment of cancer, cardiovascular diseases, infection, etc $[15,16]$. It also has cardioprotective properties, such as improving myocardial ischemia/reperfusion injury of the rats by inhibiting oxidative stress, endoplasmic reticulum stress and GSK-3 $\beta$ signal pathway [17], and improving doxorubicininduced cardiotoxicity [18]. In addation, KAE can also improve inflammatory reaction of the cardiac fibroblasts induced by LPS and ATP[19]. In addation, previous research found KAE has a protective role in fibrosis in various organs [20-22]. However, the role of KAE in AngIIinduced cardiac fibrosis has yet to be explored. The study aimed to observe the protective role of KAE in AngII induced ventricular remodelling and its possible mechanism, providing experimental basis for increasing clinical indications of KAE.

\section{Materials and Methods}

\section{Animal model}

All of the studies were performed in accordance with the Guide for the Care and Use of Laboratory Animals ( $8^{\text {th }}$ ed., National Research Council, National Academy Press, Washington, DC, 2011). After two weeks, C57BL/ 6 mice were subjected to either chronic cardiac remodelling generated by AngII infusion or saline as the control group [23]. Mice were randomly assigned into the following four groups: Saline (Sal), KAE (i.p., 10 mg/kg BW, dissolved in $100 \mu \mathrm{L}$ of PBS), AngII (1.1mg/kg/day) 2W and AngII+KAE 2W. The KAE dosage was determined according to the previous studies. To further determine whether KAE can reverse the established cardiac remodelling, the mice were randomized to receive KAE after 2-week AngII infusion, at which time cardiac remodelling can be significantly documented. The mice were randomly assigned into four groups: Sal, KAE (i.p., $10 \mathrm{mg} / \mathrm{kg} \mathrm{BW}$, dissolved in $100 \mu \mathrm{L}$ of PBS), AngII $4 \mathrm{~W}$ group and AngII 4W+KAE $2 \mathrm{~W}$ group. All of the mice in our studies were male, aged 8-10 weeks, which had body weight of 23.5-27.5 g. They were fed based on the common diet in rodent intervention studies. Ang II or saline was infused via osmotic minipumps. After the mice were killed, the heart was dissected and weighed to compare the heart 


\section{Cellular Physiology Cell Physiol Biochem 2017;43:2253-2263 \begin{tabular}{l|l|l} 
and BOI: 10.1159/000484304 & $\begin{array}{l}\text { @ } 2017 \text { The Author(s). Published by S. Karger AG, Basel } \\
\text { www.karger.com/cpb }\end{array}$
\end{tabular}}

Liu et al.: KAE Inhibits Cardiac Fibrosis

weight/body weight (HW/BW, mg/g), heart weight/tibial length (HW/TL, mg/mm) and lung weight/body weight (LW/BW, $\mathrm{mg} / \mathrm{g})$ ratios.

\section{Echocardiographic analyses}

Mice were anaesthetized with pentobarbital sodium $(6 \mathrm{mg} / \mathrm{kg}$, i.p.) for cardiac echocardiography using an ultrasound machine (Vivid 7, GE Medical System, Milwaukee, WI, USA). The left ventricular ejection fraction (EF), interventricular Septal Thickness at Diastole (IVSD), left ventricular end-systolic dimension (LVESD), left ventricular end-diastolic dimension (LVEDD) were calculated from the M-mode recording.

\section{Quantitative Real-Time PCR and Western Blot}

Total RNA was extracted using TRIZOL reagent as previously described [23], which was reversely transcribed. The first-strand cDNA was used for quantitative real-time PCR to quantify mRNA expression with GAPDH as a normalized control.

Heart tissues, cultured human umbilical vein endothelial cells (HUVECs) were lysed in RIPA buffer. Proteins were isolated as previously described [24], and the lysates (50 $\mu \mathrm{g}$ ) were subjected to electrophoresis in $10 \%$ SDS-PAGE and transferred to PVDF membrane, followed by incubation with specific antibodies against different antigens overnight at $4{ }^{\circ} \mathrm{C}$, including p-p38, p38, p-extracellular signal regulated kinases 1/2 (p-ERK 1/2), ERK 1/2, p-c-Jun N-terminal kinase (p-JNK), JNK, p-smad2, smad2, p-smad3, smad3, CD31, $\alpha$-SMA, vimentin, and GAPDH. Then the membranes were incubated with a secondary antibody and scanned by Odyssey Imaging System (LI-COR, Inc., Lincoln, NE, USA).

\section{Histological analysis and immunofluorescence}

Several heart sections (5 $\mu \mathrm{M}$ thick) were prepared as previously described [24]. The cardiomyocyte histopathology was detected using haematoxylin\&eosin (HE) staining, and collagen production was detected using picrosirius red (PSR). All of the results were visualized and photographed via light microscopy (Eclipse8, Nikon, Tokyo, Japan) and the images were analysed with ImagePro Plus software (Media Cybernetics, Bethesda, MD, USA) to quantify cardiomyocyte area and collagen percentage.

Immunofluorescent staining was performed to determine CD31 and vimentin. Briefly, the sections were autoclaved for antigen retrieval, blocked with $10 \%$ goat serum and incubated with both of the two primary antibodies overnight at $4{ }^{\circ} \mathrm{C}$. The secondary antibody was added and incubated for $1 \mathrm{~h}$. The sections were subsequently examined and photographed by a fluorescence microscope.

\section{Cultured neonatal rat cardiac fibroblasts ( $\mathrm{CFS}$ )}

CFs were prepared from the heart of the newborn (0-7 day old) Sprague-Dawley rat pups (100-150 g) as described [24], and grew in DMEM/F12 (Hyclone 8113319) medium supplemented with 20\% fetal bovine serum (FBS, GIBCO, 10099) and 1\% Penicillin Streptomycin (PS, GIBCO 1308300) at $37^{\circ} \mathrm{C}$ in a humid environment containing $5 \% \mathrm{CO}_{2}$ (SANYO 18M). The purity of the CFs was assessed by cell morphology observations and positive staining of Vimentin and $\alpha$-SMA. Cells in this study were of passage 2 to 3 . When density of the CFs reached $80 \%$ confluence, they were digested with $1 \mathrm{ml}$ 0.25\% Trypsin-EDTA (GIBCO, 1316929) and passaged according to the ratio of 1:2. Before treating the cells with different irritant, they were starved from serum for $24 \mathrm{~h}$ in order to eliminate the influence of FBS, which could make all these cells synchronized.

The toxicity of KAE on CFs was determined by the trypan blue exclusion test. Cell proliferation was determined using a non-radioactive colorimetric Cell Counting Kit CCK-8 (Dojindo, ER612) assay according to the manufacturer's instructions. Finally, DNA synthesis was measured by incorporation of BrdU using a cell proliferation ELISA kit.

Phalloidin was used to staining cytoskeletal proteins. Briefly, after treated with the corresponding protocol, CFs cells were washed twice using phosphate-buffered saline (PBS) and fixed with $4 \%$ formaldehyde for $20 \mathrm{~min}$. They were then incubated with $5 \mu \mathrm{g} / \mathrm{ml}$ phalloidin for $40 \mathrm{~min}$. After that, the coverslips were washed with PBS and mounted on glass slides with 4', 6-diamidino-2-phenylindole (DAPI), micrographs were obtained using a fluorescence microscope (Olympus, Tokyo, Japan).

Culture of HUVEC cells

The HUVEC cell line was purchased from YRGene (NC006). The cells were cultured and prepared as described previously [24]. Immunofluorescent staining was performed to determine CD31 and vimentin. 
Briefly, cells were permeabilized with 0.2\% Triton X-100 (0694, Amresco, Penn, USA) for 5 min, blocked with $10 \%$ goat serum and incubated with primary antibodies against CD31 (ab28364, ABCAM, San Diego, USA) and vimentin (ab8978, ABCAM) overnight at $4{ }^{\circ} \mathrm{C}$. The secondary antibody was added and incubated for $1 \mathrm{~h}$. The sections were subsequently examined and photographed by a fluorescence microscope.

\section{Statistical analysis}

All data were expressed as means \pm SEM. Comparisons between two groups were performed using the unpaired Student's t test. Differences among groups were determined by one-way ANOVA followed by Student-Newman-Keuls (SNK) tests. $p<0.05$ indicate a statistically difference.

\section{Results}

KAE attenuates the development of cardiac hypertrophy and dysfunction induced by AngII

Chronic AngII infusion for 2 weeks caused significant hypertrophy in the C57BL/6 mice. The HW/BW, HW/TL ratios were significantly decreased in the AngII+KAE $2 \mathrm{~W}$-treated mice compared to the AngII $2 \mathrm{~W}$-treated mice (Fig. 1A). The gross heart characteristics and the HE

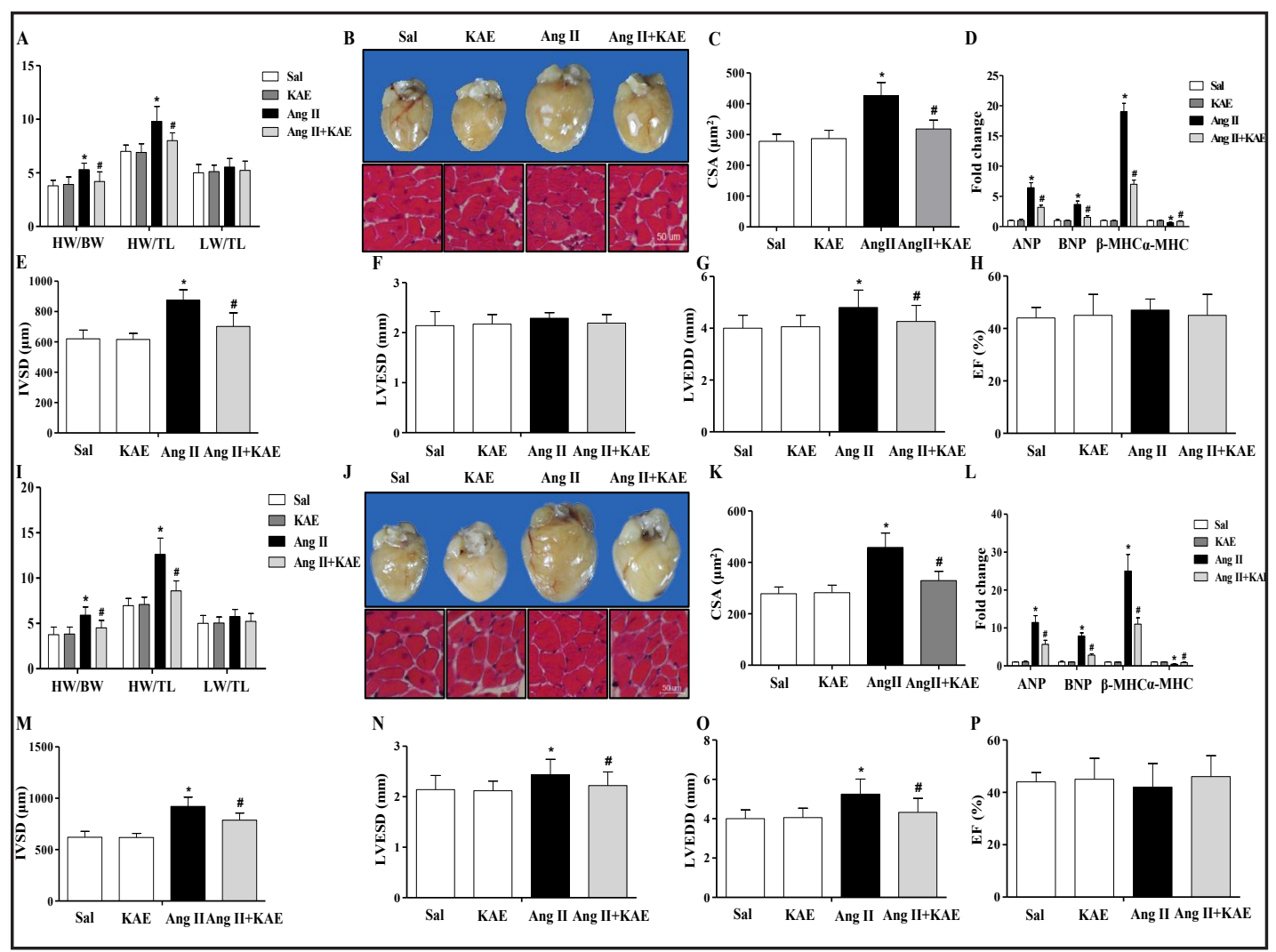

Fig. 1. The effect of KAE on AngII-induced cardiac hypertrophy and dysfunction. Statistical results of the HW/BW, HW/TL and LW/BW ratios $(\mathrm{n}=10)$ at 2 weeks after AngII and/or KAE infusion (A); the gross heart, HE staining (B) and CSA ( $n=100$ cells per section) (C) at 2 weeks after AngII and/or Sal infusion, RT-PCR analysis of hypertrophic markers including ANP, BNP, $\beta$-MHC and $\alpha$-MHC from the heart of different groups $(n=9)(D)$; echocardiography results 2 weeks after AngII and/or Sal infusion ( $n=10)(E-H)$; statistical results of the HW/BW, HW/TL and LW/BW ratios $(n=10)$ at 4 weeks after AngII and/or KAE infusion in reverse experiments (I); the gross heart, HE staining (J) and CSA ( $n=100$ cells per section) (K) at 4 weeks after AngII and/or KAE infusion, RT-PCR analysis of hypertrophic markers including ANP, BNP, $\beta$-MHC and $\alpha$-MHC from the heart of different groups $(n=9)$ in reverse experiments (L); echocardiography results 4 weeks after AngII and/or saline infusion $(n=10)(M-P)$ in reverse experiments. ${ }^{*} \mathrm{P}<0.01$, compared to Sal group; $\# \mathrm{P}<0.01$, compared to AngII group.

\section{KARGER}


staining results of cardiomyocyte cross-sectional areas (CSA) also confirmed the role of KAE in AngII-induced cardiac remodeling (Fig. 1B-C). Subsequent analysis on mRNA expression levels of atrial natriuretic peptide (ANP), B-type natriuretic peptide (BNP), $\beta$-myosin heavy chain $(\beta-\mathrm{MHC})$ and $\alpha$-myosin heavy chain $(\alpha-\mathrm{MHC})$, which were responsible for cardiac hypertrophy, showed similar trend (Fig. 1D). Echocardiographic analysis was also utilized to evaluate cardiac structures and function, and AngII+KAE $2 \mathrm{~W}$ group had significantly lower IVSD and LVEDD than Ang II 2W infusion group, without significant difference in LVESD and EF (Fig. 1E-H).

To further determine whether KAE can reverse the established cardiac hypertrophy, AngII-induced mice were randomized to receive KAE after 2-week AngII infusion, at which time cardiac hypertrophy can be significantly documented. The mice treated with KAE were compared with Sal- or AngII- treated mice at 4 weeks. Fig. 1I-L shows a significant reversal of cardiac hypertrophy in the AngII $4 \mathrm{~W}+\mathrm{KAE} 2 \mathrm{~W}$ group compared with AngII $4 \mathrm{~W}$ group. The results of echocardiogram showed that AngII $4 \mathrm{~W}+\mathrm{KAE} 2 \mathrm{~W}$ group had significantly lower IVSD, LVEDD and LVESD than Ang II $4 \mathrm{~W}$ infusion group, without significant difference in EF (Fig. 1M-P).

\section{KAE attenuates cardiac fibrosis induced by AngII}

Fibrosis contributes to the phenotypic changes associated with the development of pathological cardiac hypertrophy [25-27]. Chronic AngII infusion for 2 weeks caused significant fibrosis in C57BL/6 mice, and the PSR staining revealed that AngII-induced cardiac fibrosis (perivascular and interstitial fibrosis) was significantly attenuated in the AngII+KAE

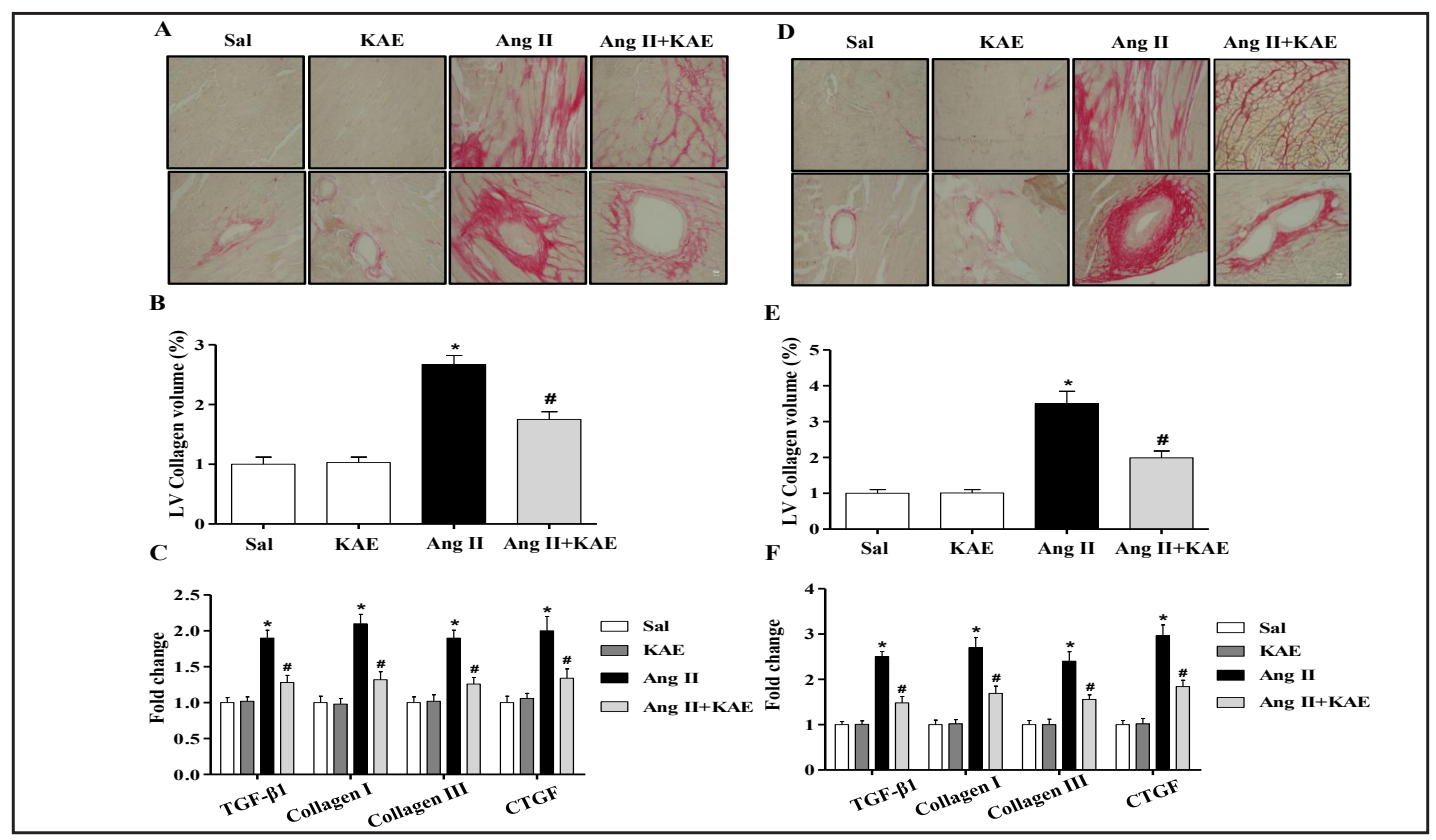

Fig. 2. The effect of KAE on AngII-induced cardiac fibrosis. Histological sections of the heart were stained for PSR in the indicated groups at 2 weeks after AngII and/or KAE infusion (A); the left ventricular interstitial collagen volume fraction in the indicated groups was quantified using an image-analyzing system (B); RT-PCR analysis on the mRNA expression of TGF- $\beta 1$, CollagenI, Collagen III and CTGF in the myocardium obtained from indicated groups at 2 weeks after AngII and/or KAE infusion (n=9) (C); histological sections of the heart were stained for PSR in the indicated groups at 4 weeks after AngII and/or KAE infusion in reverse experiments (D); left ventricular interstitial collagen volume fraction in the indicated groups was quantified using an image-analyzing system (E); RT-PCR analysis on the mRNA expression of TGF- $\beta 1$, collagen I, Collagen III and CTGF in the myocardium obtained from indicated groups at 4 weeks after AngII and/or KAE infusion ( $\mathrm{n}=9$ ) (F) in reverse experiments. ${ }^{*} \mathrm{P}<0.01$, compared to Sal group; \#P $<0.01$, compared to AngII group.

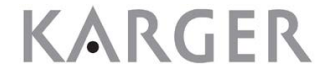




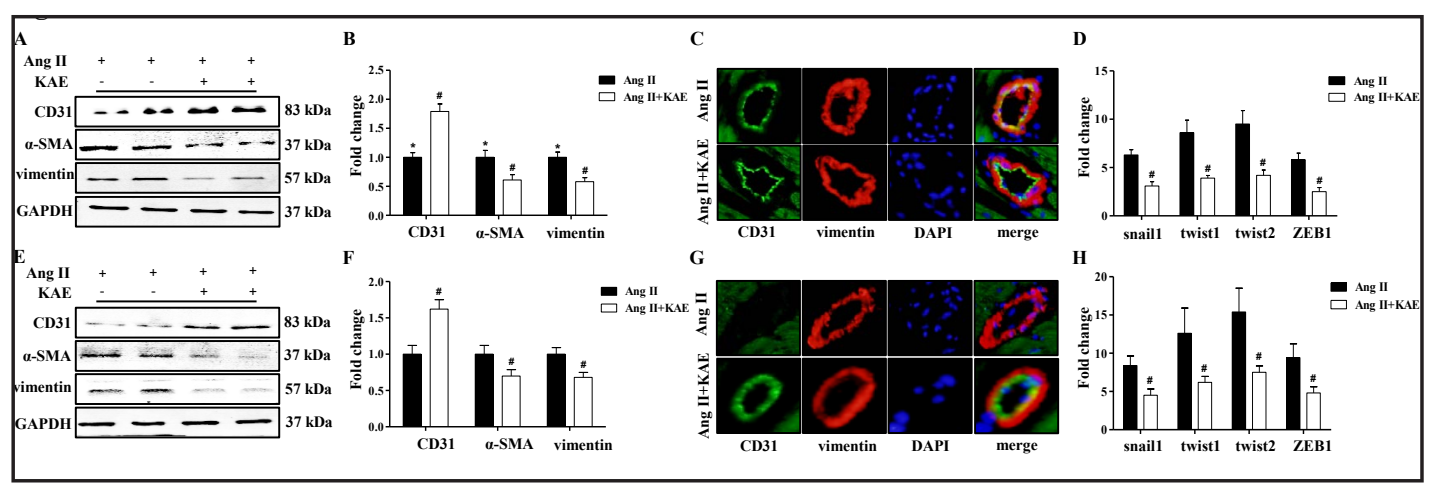

Fig. 3. The effect of KAE on AngII-induced EndMT. Representative WBs and quantitative WB analysis showed the mean CD31, $\alpha$-SMA and vimentin levels in the cardiac homogenates from the 2 groups of mice 2 weeks after AngII/AngII+KAE infusion (n=3) (A-B); myocardial sections were co-immunostained for CD31 (green)/ vimentin (red) and imaged via fluorescence microscopy (C), RT-PCR analysis on the mRNA expression of snail1, twist1, twist2 and ZEB1 in the myocardium obtained from indicated groups at 2 weeks after AngII/ AngII+KAE infusion ( $n=9$ ) (D); representative WBs and quantitative WB analysis showed the mean CD31, $\alpha$-SMA and vimentin levels in the cardiac homogenates from the 2 groups of mice 4 weeks after AngII/ AngII+KAE infusion in reverse experiments $(n=3)(E-F)$; myocardial sections were co-immunostained for CD31 (green)/vimentin (red) and imaged via fluorescence microscopy (G), RT-PCR analysis on the mRNA expression of snail1, twist1, twist2 and ZEB1 in the myocardium obtained from indicated groups at 4 weeks after AngII/AngII+KAE infusion in reverse experiments $(n=9)(H)$. $\# \mathrm{P}<0.01$, compared to AngII group.

$2 \mathrm{~W}$ group (Fig. 2A-B). Subsequent analysis on mRNA expression levels of transforming growth factor- $\beta 1$ (TGF- $\beta 1$ ), Collagen I, Collagen III and connective tissue growth factor (CTGF), which were responsible for cardiac fibrosis, showed the similar results (Fig. 2C).

To further determine whether KAE can reverse the established cardiac fibrosis, the mice treated with KAE were compared with Sal- or AngII- treated mice at 4 weeks. Fig. 2D-E shows a significant reversal of cardiac fibrosis in the AngII 4W+KAE 2W group compared with AngII 4W group. The mRNA expression of TGF- $\beta 1$, Collagen I, Collagen III and CTGF also showed similar trend (Fig. 2F). These data suggested that KAE attenuated and reverted cardiac fibrosis.

\section{KAE inhibits the EndMT induced by AngII}

Since EndMT played an important role in cardiac fibrosis during chronic AngII infusion, we investigated whether KAE attenuated cardiac fibrosis via the inhibition of the EndMT. 2 weeks after AngII infusion, WB results revealed that the heart of AngII+KAE 2W-treated mice had significantly lower levels of $\alpha$-smooth muscle actin ( $\alpha$-SMA) and vimentin compared to AngII-treated mice, while CD31 levels were significantly higher (Fig. 3A-B). These findings were also confirmed via the co-localization of CD31 and vimentin (Fig. 3C). To further elucidate the effect of KAE on the EndMT during AngII infusion, we examined certain transcription factors known to play an important role in EndMT, including snail1, twist1, twist2, and ZEB1, all of which were decreased in the AngII+KAE 2W-treated group compared with the AngII 2W-treated group (Fig. 3D).

To further determine whether KAE can reverse the established EndMT, the mice treated with KAE were compared with Sal- or AngII- treated mice at 4 weeks. WB results revealed that the heart of AngII 4W+KAE $2 \mathrm{~W}$-treated mice had significantly lower $\alpha$-SMA and vimentin compared to AngII 4W-treated mice, while CD31 levels were significantly higher (Fig. 3E-F), consistent with the results of previous studies. Co-localization of CD31 and vimentin (Fig. $3 \mathrm{G}$ ) also showed a significant reversal of EndMT in the AngII 4W+KAE $2 \mathrm{~W}$ group compared with AngII 4W group. The mRNA expression of snail1, twist1, twist2 and ZEB1 also showed the similar results (Fig. 3H). These data suggested that KAE inhibited EndMT induced by AngII.

\section{KARGER}




\section{KAE inhibits the EndMT of HUVEC cells induced by TGF- $\beta 1$}

Treated the HUVEC cells with TGF- $\beta 1(10 \mathrm{ng} / \mathrm{ml})$ for 3 consecutive days can cause transition of the endothelial cells into mesenchymal cells. As shown in Fig. 4A, KAE (5, $20,50 \mu \mathrm{M})$ and/or TGF- $\beta 1$ had no cytotoxicity on HUVEC cells. After incubation for $3 \mathrm{~d}$, TGF- $\beta 1$ significantly increased the mRNA levels of Collagen I, III, $\alpha$-SMA and fibronectin and decreased CD31, while KAE blocked EndMT induced by TGF- $\beta 1$, which was associated with the inhibition of the mesenchymal markers as well as an increase in the mRNA expression of CD31 in a dose-dependent manner (Fig. 4B). The HUVEC cells in the control group were arranged into a cobblestone-like structure, which were closely connected to each other by tight junction [28]. After stimulation with TGF- $\beta 1$ for $3 \mathrm{~d}$, the cells were gradually transited to a fusiform structure, which were loosely connected to each other by large junction. Whereas, KAE $(50 \mu \mathrm{M})$ significantly suppressed TGF- $\beta 1$ induced morphology changes (Fig. 4C). It was also confirmed by double immunofluorescence, TGF- $\beta 1$ treatment alone significantly decreased the expression level of CD31 and increased the expression of mesenchymal marker vimentin, while co-treatment with KAE $(50 \mu \mathrm{M})$ significantly reversed the phenomena (Fig. 4D). KAE alone had few effects on HUVEC cells in the above experiments.

We further explored the molecular mechanisms by which KAE inhibited the EndMT. Given that the mitogen-activated protein kinase (MAPK) signaling pathway has been previously shown to play an important role in cardiac fibrosis, we first examined whether KAE affected the TGF- $\beta 1$-induced MAPK signaling response. As expected, we observed that ERK1/2, c-Jun N-terminal kinase 1/2 (JNK1/2), and p38 were significantly phosphorylated in TGF- $\beta 1$-induced group. However, the phosphorylation of p38 was almost completely inhibited in the TGF- $\beta 1+$ KAE group compared with the TGF- $\beta 1$ alone group, whereas the phosphorylation of ERK1/2 and JNK1/2 were not significantly affected. Furthermore, we assessed the regulatory role of KAE on Smad cascade activation. Our results showed TGF- $\beta 1$ induced increased levels of Smad 2/3 phosphorylation were significantly attenuated in TGF$\beta 1+\mathrm{KAE}$ group compared with TGF- $\beta 1$ alone group. At the same time, the expression of total protein did not change either in TGF- $\beta 1$ group or TGF- $\beta 1+$ KAE group. Snail 1 , twist1, twist 2 and ZEB1 which played an important role in EndMT, our results showed their expressions were all up-regulated in TGF- $\beta 1$ group, whereas KAE significantly blocked the upregulation

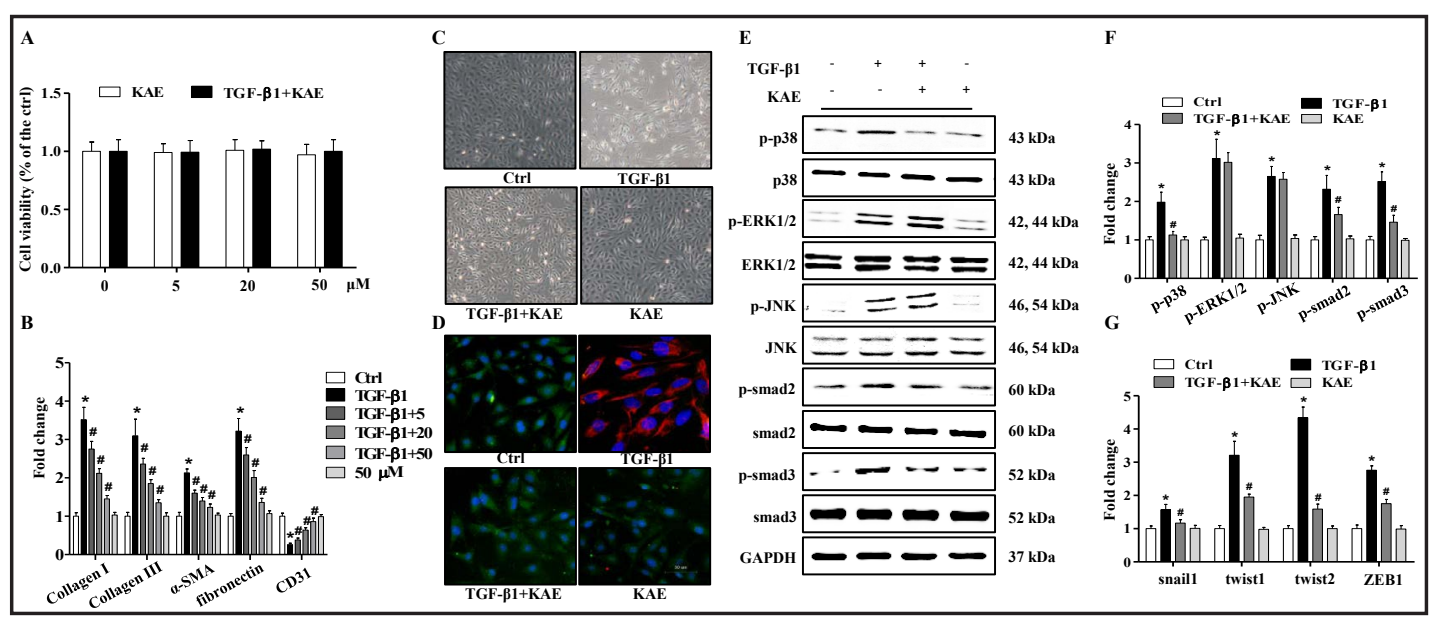

Fig. 4. KAE ameliorated EndMT in HUVEC cells. Cell viability was examined by trypan blue exclusion test (A); RT-PCR analysis on the mRNA expression of Collagen I, Collagen III, $\alpha$-SMA, fibronectin and CD31 in the HUVEC cells from indicated groups at 3 days after TGF- $\beta 1 /$ KAE treatment $(n=6)(B)$; the morphology change of HUVEC was observed under the light microscope (C); HUVEC cells were co-immunostained for CD31 (green)/vimentin (red) and imaged via fluorescence microscopy (D); representative WBs and quantitative WB analysis showed the mean protein levels in the cells from the 4 groups at 3 days after TGF- $\beta 1 /$ KAE treatment $(n=3)(E-F)$ and the mRNA expression levels of snail1, twist1, twist2 and ZEB1 in the cells were determined in via RT-PCR $(n=6)(G) .{ }^{*} \mathrm{P}<0.01$, compared to Ctrl group; \#P $<0.01$, compared to TGF- $\beta 1$ group. 


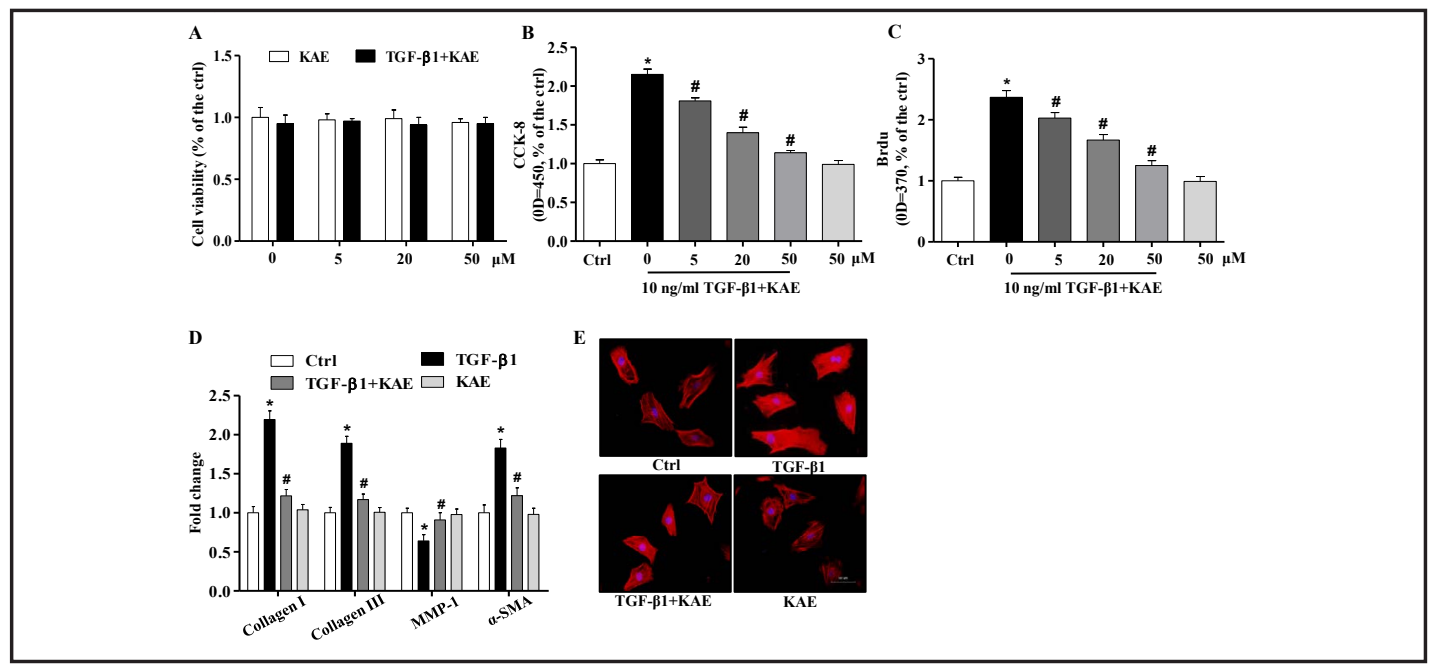

Fig. 5. The effect of KAE on the proliferation of CFs induced by TGF- $\beta 1$. Cell viability was examined by trypan blue exclusion test (A); cell proliferation was examined by CCK-8 (B); Brdu incorporation was also determined with an ELISA-based assay (C); RT-PCR analysis on the mRNA expression of Collagen I, Collagen III, MMP- 1 and $\alpha$-SMA in the CFs from indicated groups at $24 \mathrm{~h}$ after TGF- $\beta 1 /$ KAE treatment (n=6) (D); CFs were immunostained for phalloidin and imaged via fluorescence microscopy (E). ${ }^{*} \mathrm{P}<0.01$, compared to Ctrl group; \#P<0.01, compared to TGF- $\beta 1$ group.

of the studied transcription factors induced by TGF- $\beta 1$ (Fig. 4E-G). Together, these results demonstrate that the detrimental role of KAE in EndMT is largely associated with the regulation of p38 and smad $2 / 3$ signaling.

\section{KAE blocks the proliferation of CFs induced by TGF- $\beta 1$}

Different concentrations of $\operatorname{KAE}(5,20,50 \mu \mathrm{M})$ and/or TGF- $\beta 1$ had no cytotoxicity on CFs (Fig. 5A). To determine the effect of KAE on CFs proliferation, the CFs were treated with different concentrations of $\operatorname{KAE}(5,20,50 \mu \mathrm{M})$ with TGF- $\beta 1$ for $24 \mathrm{~h}$ using the CCK-8 cell proliferation assay. Compared with the control group, TGF- $\beta 1$ significantly induced CFs proliferation, which was blocked by KAE in a concentration-dependent manner (Fig. 5B). It was also confirmed by the inhibitory effect of DNA synthesis by measuring the incorporation of BrdU (Fig. 5C), and the mRNA expression of Collagen I, Collagen III, MMP-1 and $\alpha$-SMA also showed similar results (Fig. 5D). The effects of KAE on F-action polymerization were further investigated. As shown by phalloidin fluorescent in Fig. 5E, increased actin stress fiber formation was observed when the CFs cells were treated with TGF- $\beta 1$ for up to $24 \mathrm{~h}$, and this reorganization caused by TGF- $\beta 1$ was suppressed by KAE $(50 \mu \mathrm{M})$, while KAE alone had few effects on CFs in the above experiments.

\section{Discussion}

The KAE in this model had no basal effects but attenuated the cardiac hypertrophy, cardiac dysfunction and fibrosis induced by AngII infusion. We also found that KAE effectively attenuated interstitial fibrosis by inhibiting EndMT and CFs proliferation as well as collagen production, all of which contributed to the development of cardiac fibrosis. All of the above results suggest that KAE inhibits endothelial dysfunction and activation, which may be an important mechanism that attenuates cardiac fibrosis and left ventricular diastolic dysfunction in pathologic condition of AngII infusion. Our results indicate that KAE might be a novel therapy for preventing cardiac remodelling diseases.

Cardiac endothelial cells play an important role in maintaining vascular tone and haemostasis and in regulating coagulation [29]. However, endothelial dysfunction 
induced by various factors such as smoking, hyperlipidaemia and oxidative stress may cause cardiovascular disease. In recent years, EndMT is becoming a hotspot of researchers, as the gradual study into the development mechanism of organ fibrosis. When organ fibrosis develops to a certain stage, endothelial cells in small vessels are gradually converted to fibroblasts with active synthesis and secretion, which are then converted to myofibroblasts, accomplishing EndMT. In 2007, Zeisberg et al [6] found endothelial cells were converted to fibroblasts via EndMT during the myocardial fibrosis of mice induced by pressure overload, which promoted myocardial fibrosis. Cardiac function can be dramatically improved by inhibiting EndMT, indicating that EndMT played an important role in myocardial fibrosis. Thus, exploring EndMT and finding the key factors which regulated EndMT were significant for the target selection of clinical treatment.

We found that the EndMT was significantly decreased in AngII+KAE-treated group compared with the AngII-treated group. Additionally, the gene expression levels that played an important role in promoting the EndMT were decreased in the AngII+KAE-treated group. The in vitro experiment also confirmed that KAE significantly inhibited the EndMT of HUVEC cells induced by TGF- $\beta 1$. These results strongly suggest that KAE plays an important role in attenuating the EndMT, inhibiting cardiac fibrosis in response to AngII infusion.

TGF- $\beta 1 /$ Smads and MAPKs signal pathways as well as some downstream transcription factors, including snail, twist and ZEB family, played an important regulatory role in cardiac EndMT[24, 30]. In the study, the expression levels of MAPKs and Smads signal pathways as well as their downstream transcription factors were detected. The in vitro experiment found that phosphorylation levels of p38 and smad 2/3 signal pathways were dramatically increased, and expression levels of the downstream target genes snail1, twist1, twist2 and ZEB1 were clearly up-regulated after HUVEC cells were stimulated by TGF- $\beta 1$ for 24 $h$. However, expression levels of the above mentioned signal pathways and transcription factors were dramatically down-regulated when both TGF- $\beta 1$ and KAE were used. Therefore, it is considered that KAE can suppress EndMT of HUVEC cells via inhibiting the expression of p38 and smad 2/3 signal pathways as well as their downstream transcription factors.

In our model, cardiac remodelling gradually developed, and partial vascular endothelial cells were gradually changed to mesenchymal fibroblasts after AngII infusion for $2 \mathrm{~W}$. The vascular endothelial cells during the early stage not only had features of endothelial cells but also had characteristics of mesenchymal cells, which were positive for CD31/ $\alpha$-SMA staining. Under continuous infusion of AngII, partial endothelial cells completely lost features of endothelial cells at $4 \mathrm{~W}$, which only showed characteristics of mesenchymal cells. At this time, CD31 fluorescent staining showed negative results and only features of mesenchymal cells were shown.

The previous studies demonstrated that the binding of AngII to AT1 receptors on fibroblasts can stimulate proliferation of fibroblasts and deposition of collagen in ECM, and subsequently cause myocardial fibrosis [31]. The current study also confirmed that KAE can lower cellular proliferation induced by AngII and synthesis and expression of type I and III collagen protein, increasing expression of MMP-1. Hence, it is thought that KAE can decrease myocardial fibrosis by inhibiting proliferation and activation of the cardiac fibroblasts.

There are also some limitations in the study. Whether KAE can decrease myocardial hypertrophy directly is not clear, and the effects of KAE on the other risk factors inducing ventricular remodelling, such as inflammation, oxidative stress, apoptosis and autophagy are also not clear. The effects of KAE on the factors above will be further explored in the future. Besides, the current study also provides experimental basis for clinical application of KAE.

\section{Acknowledgements}

Conceived and designed the experiments: Yuan Liu and Haibo Yang. Performed experiments: Ling Li, Xiaoyan Zhao, Yanzhou Zhang and Xiaofei Yan. Analyzed the data: Sen 


\section{Cellular Physiology Cell Physiol Biochem 2017;43:2253-2263 \begin{tabular}{ll|l} 
DOI: 10.1159/000484304 & $\begin{array}{l}\text { C } 2017 \text { The Author(s). Published by S. Karger AG, Basel } \\
\text { www.karger.com/cpb }\end{array}$ \\
\hline
\end{tabular}}

Liu et al.: KAE Inhibits Cardiac Fibrosis

Guo, Yuzhou Liu and Shuai Wang. Contributed reagents/materials/analysis tools: Lu Gao and Yunpeng. Wrote the paper: Yuan Liu. All authors read and approved the final manuscript. We would like to thank all the participants for their support.

This work was supported by a grant from the scientific and technological project of Henan province (172102310531).

\section{Disclosure Statement}

The authors declare that there is no Disclosure Statements regarding the publication of this paper.

\section{References}

1 Sirish P, Li N, Liu JY, Lee KS, Hwang SH, Qiu H, Zhao C, Ma SM, Lopez JE, Hammock BD, Chiamvimonvat $\mathrm{N}$ : Unique mechanistic insights into the beneficial effects of soluble epoxide hydrolase inhibitors in the prevention of cardiac fibrosis. Proc Natl Acad Sci U S A 2013;110:5618-5623.

2 Hong Y, Cao H, Wang Q, Ye J, Sui L, Feng J, Cai X, Song H, Zhang X, Chen X: MiR-22 may Suppress Fibrogenesis by Targeting TGFßR I in Cardiac Fibroblasts. Cell Physiol Biochem 2016;40:1345-1353.

- 3 Porter KE, Turner NA: Cardiac fibroblasts: at the heart of myocardial remodeling. Pharmacol Ther 2009;123:255-278.

4 Wang K, Lu D, Zhang B, Wang S, Liu Q, Zhang Q, Geng J, Shan Q: Renal Denervation Attenuates MultiOrgan Fibrosis and Improves Vascular Remodeling in Rats with Transverse Aortic Constriction Induced Cardiomyopathy. Cell Physiol Biochem 2016;40:465-476.

5 Santiago JJ, Dangerfield AL, Rattan SG, Bathe KL, Cunnington RH, Raizman JE, Bedosky KM, Freed DH, Kardami E, Dixon IM: Cardiac fibroblast to myofibroblast differentiation in vivo and in vitro: expression of focal adhesion components in neonatal and adult rat ventricular myofibroblasts. Dev Dyn 2010;239:15731584.

6 Zeisberg EM, Tarnavski O, Zeisberg M, Dorfman AL, McMullen JR, Gustafsson E, Chandraker A, Yuan X, Pu WT, Roberts AB, Neilson EG, Sayegh MH, Izumo S, Kalluri R: Endothelial-to-mesenchymal transition contributes to cardiac fibrosis. Nat Med 2007;13:952-961.

7 Kolditz DP, Wijffels MC, Blom NA, van der Laarse A, Hahurij ND, Lie-Venema H, Markwald RR, Poelmann RE, Schalij MJ, Gittenberger-de GAC: Epicardium-derived cells in development of annulus fibrosis and persistence of accessory pathways. Circulation 2008;117:1508-1517.

8 van Amerongen MJ, Bou-Gharios G, Popa E, van Ark J, Petersen AH, van Dam GM, van Luyn MJ, Harmsen MC: Bone marrow-derived myofibroblasts contribute functionally to scar formation after myocardial infarction. J Pathol 2008;214:377-386.

-9 Haudek SB, Xia Y, Huebener P, Lee JM, Carlson S, Crawford JR, Pilling D, Gomer RH, Trial J, Frangogiannis NG, Entman ML: Bone marrow-derived fibroblast precursors mediate ischemic cardiomyopathy in mice. Proc Natl Acad Sci U S A 2006;103:18284-18289.

10 Diaz-Flores L, Gutierrez R, Madrid JF, Varela H, Valladares F, Acosta E, Martin-Vasallo P, Diaz-Flores L Jr: Pericytes. Morphofunction, interactions and pathology in a quiescent and activated mesenchymal cell niche. Histol Histopathol 2009;24:909-969.

-11 Widyantoro B, Emoto N, Nakayama K, Anggrahini DW, Adiarto S, Iwasa N, Yagi K, Miyagawa K, Rikitake Y, Suzuki T, Kisanuki YY, Yanagisawa M, Hirata K: Endothelial cell-derived endothelin-1 promotes cardiac fibrosis in diabetic hearts through stimulation of endothelial-to-mesenchymal transition. Circulation 2010;121:2407-2418.

12 Murdoch CE, Chaubey S, Zeng L, Yu B, Ivetic A, Walker SJ, Vanhoutte D, Heymans S, Grieve DJ, Cave AC, Brewer AC, Zhang M, Shah AM: Endothelial NADPH oxidase-2 promotes interstitial cardiac fibrosis and diastolic dysfunction through proinflammatory effects and endothelial-mesenchymal transition. J Am Coll Cardiol 2014;63:2734-2741.

13 Lee YJ, Choi HS, Seo MJ, Jeon HJ, Kim KJ, Lee BY: Kaempferol suppresses lipid accumulation by inhibiting early adipogenesis in 3T3-L1 cells and zebrafish. Food Funct 2015;6:2824-2833. 


\section{Cellular Physiology Cell Physiol Biochem 2017;43:2253-2263 \begin{tabular}{l|l|l} 
and Biochemistry $10.1159 / 000484304$ & $\begin{array}{l}\text { C) } 2017 \text { The Author(s). Published by S. Karger AG, Basel } \\
\text { www.karger.com/cpb }\end{array}$ \\
\hline
\end{tabular}}

Liu et al.: KAE Inhibits Cardiac Fibrosis

14 Hoang MH, Jia Y, Mok B, Jun HJ, Hwang KY, Lee SJ: Kaempferol ameliorates symptoms of metabolic syndrome by regulating activities of liver X receptor- $\beta$. J Nutr Biochem 2015;26:868-875.

15 Rajendran P, Rengarajan T, Nandakumar N, Palaniswami R, Nishigaki Y, Nishigaki I: Kaempferol, a potential cytostatic and cure for inflammatory disorders. Eur J Med Chem 2014;86:103-112.

-16 Devi KP, Malar DS, Nabavi SF, Sureda A, Xiao J, Nabavi SM, Daglia M: Kaempferol and inflammation: From chemistry to medicine. Pharmacol Res 2015;99:1-10.

-17 Zhou M, Ren H, Han J, Wang W, Zheng Q Wang D: Protective Effects of Kaempferol against Myocardial Ischemia/Reperfusion Injury in Isolated Rat Heart via Antioxidant Activity and Inhibition of Glycogen Synthase Kinase-3ß. Oxid Med Cell Longev 2015;2015:481405.

$>18$ Xiao J, Sun GB, Sun B, Wu Y, He L, Wang X, Chen RC, Cao L, Ren XY, Sun XB: Kaempferol protects against doxorubicin-induced cardiotoxicity in vivo and in vitro. Toxicology 2012;292:53-62.

19 Tang XL, Liu JX, Dong W, Li P, Li L, Hou JC, Zheng YQ, Lin CR, Ren JG: Protective effect of kaempferol on LPS plus ATP-induced inflammatory response in cardiac fibroblasts. Inflammation 2015;38:94-101.

20 Li H, Yang L, Zhang Y, Gao Z: Kaempferol inhibits fibroblast collagen synthesis, proliferation and activation in hypertrophic scar via targeting TGF- $\beta$ receptor type I. Biomed Pharmacother 2016;83:967-974.

21 Feng H, Cao J, Zhang G, Wang Y: Kaempferol Attenuates Cardiac Hypertrophy via Regulation of ASK1/MAPK Signaling Pathway and Oxidative Stress. Planta Med 2017;83:837-845.

22 Gong JH, Cho IH, Shin D, Han SY, Park SH, Kang YH: Inhibition of airway epithelial-to-mesenchymal transition and fibrosis by kaempferol in endotoxin-induced epithelial cells and ovalbumin-sensitized mice. Lab Invest 2014;94:297-308.

-23 Dang MQ Zhao XC, Lai S, Wang X, Wang L, Zhang YL, Liu Y, Yu XH, Liu Y, Li HH, Xia YL: Gene expression profile in the early stage of angiotensin II-induced cardiac remodeling: a time series microarray study in a mouse model. Cell Physiol Biochem 2015;35:467-476.

-24 Liu Y, Hu ZF, Liao HH, Liu W, Liu J, Ma ZG, Wu QQ Xu M, Zhang N, Zhang Y, Bian ZY, Tang QZ: Toll-like receptor 5 deficiency attenuates interstitial cardiac fibrosis and dysfunction induced by pressure overload by inhibiting inflammation and the endothelial-mesenchymal transition. Biochim Biophys Acta 2015;1852:2456-2466.

-25 Yang J, Chen YN, Xu ZX, Mou Y, Zheng LR: Alteration of RhoA Prenylation Ameliorates Cardiac and Vascular Remodeling in Spontaneously Hypertensive Rats. Cell Physiol Biochem 2016;39:229-241.

26 Ju CH, Wang XP, Gao CY, Zhang SX, Ma XH, Liu C: Blockade of KCa3.1 Attenuates Left Ventricular Remodeling after Experimental Myocardial Infarction. Cell Physiol Biochem 2015;36:1305-1315.

-27 Liu L, Wang C, Sun D, Jiang S, Li H, Zhang W, Zhao Y, Xi Y, Shi S, Lu F, Tian Y, Xu C, Wang L: Calhex 231 Ameliorates Cardiac Hypertrophy by Inhibiting Cellular Autophagy in vivo and in vitro. Cell Physiol Biochem 2015;36:1597-1612.

28 Beese M, Wyss K, Haubitz M, Kirsch T: Effect of cAMP derivates on assembly and maintenance of tight junctions in human umbilical vein endothelial cells. BMC Cell Biol 2010;11:68.

29 Moore C, Sanz-Rosa D, Emerson M: Distinct role and location of the endothelial isoform of nitric oxide synthase in regulating platelet aggregation in males and females in vivo. Eur J Pharmacol 2011;651:152158.

-30 Qi HP, Wang Y, Zhang QH, Guo J, Li L, Cao YG, Li SZ, Li XL, Shi MM, Xu W, Li BY, Sun HL: Activation of peroxisome proliferator-activated receptor $\gamma$ (PPAR $\gamma$ ) through NF- $\kappa$ B/Brg1 and TGF- $\beta 1$ pathways attenuates cardiac remodeling in pressure-overloaded rat hearts. Cell Physiol Biochem 2015;35:899-912.

31 Chen K, Chen J, Li D, Zhang X, Mehta JL: Angiotensin II regulation of collagen type I expression in cardiac fibroblasts: modulation by PPAR-gamma ligand pioglitazone. Hypertension 2004;44:655-661. 